\title{
Indirect or direct restorations for heavily restored posterior adult teeth?
}

\author{
Dominic Hurst \\ Centre for Evidence-based Dentistry, Oxford, UK
}

\begin{abstract}
Clinical bottom line There is no high quality evidence that supports or rejects the practice of placing a crown or onlay on a vital posterior tooth rather than a composite or amalgam restoration to ensure longer tooth survival.
\end{abstract}

\section{Clinical scenario}

An adult patient attends with several posterior teeth heavily restored with amalgam. Her brother, with a similar degree of prior dental treatment, has just had several teeth crowned by his dentist in order to prevent them being lost due to the teeth fracturing. She asks whether she should also be considering crowns.

Several options exist for the restoration of posterior teeth where moderate to severe destruction of the tooth has taken place (two or more surfaces, including occlusal wear when facial and interproximal tooth surfaces are also lost). Whilst direct placement of restorative materials such as amalgam and composite are relatively conservative and inexpensive, the placement of crowns and cast onlays may only be possible with further loss of tooth material and/or increased financial cost.

The 1998 Adult Dental Health Survey reported that one third of the adult dentate population in the United Kingdom had at least one crown. ${ }^{1}$ But both crowns and onlays, like amalgams and composites, may fail due to disease or technical and material issues. In some cases the restoration itself or the failure of the restoration may lead to further tooth loss, irreversible pulpal damage or catastrophic tooth fracture. For a general practitioner trying to decide when to place a crown or onlay there is a dilemma: do the purported benefits of restoring a tooth with this method outweigh the harms and costs? This dilemma appears to be faced by many. ${ }^{2,3}$

\section{Question formulation}

A PICO (population/patient/problem, intervention, comparison/control, outcome)

\begin{tabular}{|c|c|c|c|c|c|}
\hline \multicolumn{2}{|c|}{ Patient/problem } & Intervention & Comparison & \multicolumn{2}{|c|}{ Outcome } \\
\hline \multicolumn{2}{|c|}{$\begin{array}{l}\text { an asymptomatic vital adult } \\
\text { posterior tooth requiring a two } \\
\text { surface or larger restoration }\end{array}$} & crown or onlay & composite or amalgam & \multicolumn{2}{|c|}{ tooth survival } \\
\hline $\begin{array}{l}\text { Search } \\
\text { number }\end{array}$ & \multicolumn{4}{|l|}{$\begin{array}{l}\text { Search } \\
\text { terms }\end{array}$} & $\begin{array}{l}\text { Retrieved } \\
\text { articles }\end{array}$ \\
\hline$\# 1$ & \multicolumn{4}{|c|}{ ("Molar"[Mesh] OR "Bicuspid"[Mesh]) OR (molar OR premolar OR bicuspid) } & 65661 \\
\hline \#2 & \multicolumn{4}{|c|}{ ("Dental Restoration, Permanent"[Mesh]) OR (amalgam OR composite) } & 86782 \\
\hline \#3 & \multicolumn{4}{|c|}{ ("Crowns"[Mesh] OR "Inlays"[Mesh]) OR (crown OR inlay OR onlay) } & 33774 \\
\hline \#4 & \multicolumn{4}{|l|}{ \#1 AND \#2 AND \#3 } & 2071 \\
\hline \#5 & \multicolumn{4}{|c|}{$\begin{array}{l}\text { \#4 AND ((randomised controlled trial[pt]) OR (controlled clinical trial[pt]) } \\
\text { OR (randomised[tiab]) OR (placebo[tiab]) OR (drug therapy[sh]) OR } \\
\text { (randomly[tiab]) OR (trial[tiab]) OR (groups[tiab])) NOT (animals[mh] } \\
\text { NOT humans[mh]) }\end{array}$} & 545 \\
\hline \#6 & \multicolumn{4}{|l|}{ \#4 Limits: Reviews } & 91 \\
\hline \#7 & \multicolumn{4}{|c|}{ \#4 Limits: Practice Guideline } & 1 \\
\hline
\end{tabular}

format was used to help frame the dilemma in a potentially answerable question: for an asymptomatic vital adult posterior tooth requiring a two surface or larger restoration (Problem), do crowns or onlays (Intervention) compared to composites or amalgams (Comparison) result in the tooth being retained longer (Outcome)? ${ }^{4}$ Restoration of worn teeth is included in this patient population as facial and interproximal surfaces as well as the occlusal are lost during tooth surface loss.

\section{Search strategy}

A search strategy was created using the above PICO. It was anticipated that the following secondary outcomes might also reasonably be reported in randomised controlled studies: loss of restoration, number of re-restorations required, financial costs, patient factors (e.g. comfort during procedure, satisfaction with restoration, time), pulpal pathology requiring endodontic treatment, wear of restoration, wear of opposing tooth, caries, periodontal disease, apical pathology (including abscess, sinus) and pain. However, to allow for studies reporting on these to be included, no outcome terms were included in the search strategy so as not to restrict the search unnecessarily.

PubMED was searched using the following combination of terms:

Search \#5 combined \#4 with the Cochrane Highly Sensitive Search Strategy for identifying randomised trials in Medline: sensitivitymaximising version (2008 revision $)^{5}$ in order to reduce the retrieved articles to a manageable 545 hits. All of these were screened along with the articles identified by searches \#6 and \#7. The search strategy was repeated for the final time on 06/09/2010. OVID Medline (1950 to August Week 3 2010), CENTRAL, 
TRIP Database and ISI Web of Knowledge were searched using a similar strategy. The National Guidelines Clearinghouse website was searched using just the term "crown". The NHS Evidence Oral Health Specialist Collection was searched using "crown".

\section{Eligibility}

Evidence-based guidelines, systematic reviews or prospective randomised clinical trials would be included. Studies would be considered that: included adult premolars or molars in patients of any age as long as the teeth (or an identifiable subset) were vital; included a crown or onlay group and a concurrent composite or amalgam group; involved restoration of two surfaces or more. There was no followup time restriction in case there were studies that reported on short term outcomes (e.g. patient comfort) and there was no language restriction.

\section{Study selection}

The titles and abstracts of all reports of studies were screened using the eligibility criteria above. Where it was uncertain whether the study met the eligibility criteria, the paper was accessed in full.

\section{Results}

There were no evidence-based clinical guidelines, systematic reviews or clinical studies that met the eligibility criteria. One critical appraisal of a review was identified ${ }^{6}$ but the review was not considered to be systematic. The review itself had already been deemed ineligible as it did not claim to be a systematic review nor did it have a search strategy or inclusion/exclusion criteria. ${ }^{7}$ The only randomised controlled study that compared crowns and composites for adult teeth was on root-filled premolars over a period of three years. ${ }^{8}$ This DEBT was only concerned with the management of vital teeth owing to the risk of one or other restoration causing loss of vitality, so the report was rejected. One prospective study compared large amalgams with crowns over a period of 5 years ${ }^{9}$ and another over a period of 17 years. ${ }^{10}$ Both were non-randomised studies with no indication that cases were controlled and were therefore not considered.

\section{Discussion}

At what point do we make the decision to incur biological, financial and time costs on a patient by providing a crown or onlay rather than a direct restoration? When do the benefits of such treatment outweigh the costs?

This DEBT was unable to find prospective randomised controlled studies or systematic reviews that compared these two interventions. Nor were there any evidence-based clinical guidelines to assist the clinician.
Thus in answer to the patient enquiring whether a crown will result in her keeping her natural teeth longer, the clinician can only say that there is no high quality clinical evidence to suggest that placing a crown on a posterior tooth would lead to its longer retention than a composite or amalgam.

1. Kelly M, Steele J, Nuttall N, et al. Artificial crowns. In Walker A, Cooper I, (eds) Adult Dental Health Survey: Oral Health in the United Kingdom 1998. London: The Stationary Office 1999.

2. Bader JD, Shugars DA, Roberson TM. Using crowns to prevent tooth fracture. Community Dent Oral Epidemiol 1996; 24: 47-51.

3. Shugars DA, Hayden WJ, Crall JJ, et al. Variation in the use of crowns and their alternatives. J Dent Educ 1997; 61: $22-28$

4. Richardson WS, Wilson MC, Nishikawa J, et al. The well-built clinical question: a key to evidence-based decisions. ACP / Club 1995; 123: A12-13.

5. Lefebvre C, Manheimer E, Glanville J. Searching for studies. In Higgins J, Green S, (eds) Cochrane Handbook for Systematic Reviews of Interventions. pp 137: Wiley 2008.

6. Goldstein GR. The longevity of direct and indirect posterior restorations is uncertain and may be affected by a number of dentist-, patient-, and material-related factors. J Evid Based Dent Pract 2010; 10: $30-31$

7. Hickel R, Manhart J. Longevity of restorations in posterior teeth and reasons for failure. J Adhes Dent 2001; 3: 45-64.

8. Mannocci F, Bertelli E, Sherriff $M$, et al. Three-year clinical comparison of survival of endodontically treated teeth restored with either full cast coverage or with direct composite restoration. / Prosthet Dent 2002; 88: 297-301.

9. Martin JA, Bader JD. Five-year treatment outcomes for teeth with large amalgams and crowns. Oper Dent 1997; 22: 72-78.

10. Van Nieuwenhuysen JP, D'Hoore W, Carvalho et al. Long-term evaluation of extensive restorations in permanent teeth. J Dent 2003; 31: 395-405.

Evidence-Based Dentistry (2010) 11, 116-117. doi:10.1038/sj.ebd. 6400760 . 